\title{
Zero Controllability Criterion for Discrete Positive Systems with Multiple Delays on Both States and Inputs
}

\author{
Mouhcine Naim \\ Laboratory of Analysis, Modeling and Simulation (LAMS), Faculty of Sciences Ben M'sik, Hassan II University, \\ P. O. Box 7955 Sidi Othman, Casablanca, Morocco
}

Correspondence should be addressed to Mouhcine Naim; naimmouhcine2013@gmail.com

Received 11 April 2021; Accepted 1 May 2021; Published 12 May 2021

Academic Editor: Niansheng Tang

Copyright (c) 2021 Mouhcine Naim. This is an open access article distributed under the Creative Commons Attribution License, which permits unrestricted use, distribution, and reproduction in any medium, provided the original work is properly cited.

Zero controllability criterion for positive linear discrete systems with multiple delays in both states and inputs is obtained and proved. An example is given to support our main result.

\section{Introduction and Preliminaries}

Throughout this paper, we use the following notation: $\mathbb{N}$ is the set of nonnegative integers, $\mathbb{N}^{*}$ is the set of positive integers, $\sigma_{n}^{m}=\{n, n+1, \ldots, m\}$ is the finite subset of $\mathbb{N}$ with $n \leq m, \mathbb{R}^{n}$ is the set of real vectors with $n$ components, $\mathbb{R}_{+}^{n}$ is the set of vectors in $\mathbb{R}^{n}$ with nonnegative components, $\mathbb{R}^{n \times m}$ is the set of real matrices of size $n \times m, I_{n}$ is the identity matrix in $\mathbb{R}^{n \times n}$, and $\mathbb{R}_{+}^{n \times m}$ is the set of real matrices with nonnegative entries.

Positive systems are a wide class of systems in which state variables are constrained to be positive or at least nonnegative for all time whenever the initial conditions and inputs are nonnegative $[1,2]$. The mathematical theory of positive linear systems is based on the theory of nonnegative matrices developed by Perron and Frobenius, see, e.g., [3, 4]. Since positive systems are not defined on linear spaces but on cones, then many concepts of linear systems cannot be directly generalized to linear positive systems without reformulation. One such property is the notion of controllability of linear positive systems.

Controllability is one of the fundamental concepts in the mathematical control theory. A positive system is controllable if it is possible to transfer it from an arbitrary nonnegative initial state to an arbitrary nonnegative final state using only certain admissible nonnegative controls. Since late 1980s, controllability of discrete positive linear systems without delays has been a subject of much research [5-9]. In particular, Coxson and Shapiro in [6] showed that the discrete linear positive system is controllable if and only if it is reachable (controllability from zero initial conditions) and zero controllable (controllability to zero final state). The reachability of positive linear discrete systems with multiple delays in both state and control is addressed in [10]. On zero controllability of positive linear discrete systems with delay, the authors of [11] show that the following system with a single state delay

$$
x_{i+1}=A_{0} x_{i}+A_{1} x_{i-1}+B u_{i}, \quad i \in \mathbb{N},
$$

is zero controllable if and only if the matrix $A=\left(\begin{array}{cc}A_{0} & A_{1} \\ I_{n} & 0\end{array}\right)$ is nilpotent. In this paper, we will extend the result of zero controllability in [11] to the more general case, namely, positive discrete systems with multiple time delays both in state and in input. For this, we consider the general discrete linear time delay systems:

$$
x_{i+1}=\sum_{j=0}^{p} A_{j} x_{i-j}+\sum_{j=0}^{q} B_{j} u_{i-j}, \quad i \in \mathbb{N},
$$

where $x_{i} \in \mathbb{R}^{n}$ is the state, $u_{i} \in \mathbb{R}^{m}$ is the input, $A_{j} \in \mathbb{R}^{n \times n}\left(j \in \sigma_{0}^{p}\right), B_{j} \in \mathbb{R}^{n \times m}\left(j \in \sigma_{0}^{q}\right)$, and $p$ and $q$ are the nonnegative integer maximal values of delays on state and 
input, respectively. The initial conditions for (2) are given arbitrarily by $x_{-j} \in \mathbb{R}^{n}$ for $j \in \sigma_{0}^{p}$ and $u_{-j} \in \mathbb{R}^{m}$ for $j \in \sigma_{1}^{q}$.

Definition 1 (positivity). System (2) is said to be positive if the state $x_{i} \in \mathbb{R}_{+}^{n}, \quad i \in \mathbb{N}$, for any initial states $x_{-j} \in \mathbb{R}_{+}^{n}\left(j \in \sigma_{0}^{p}\right)$, for any initial inputs $u_{-j} \in \mathbb{R}_{+}^{m}\left(j \in \sigma_{1}^{q}\right)$, and all inputs $u_{i} \in \mathbb{R}_{+}^{m}, i \in \mathbb{N}$.

Lemma 1 (see [10]). System (2) is positive if and only if $A_{j} \in \mathbb{R}_{+}^{n \times n}\left(j \in \sigma_{0}^{p}\right)$ and $B_{j} \in \mathbb{R}_{+}^{n \times m}\left(j \in \sigma_{0}^{q}\right)$.

In all the sequels in this paper, we assume that system (2) is positive.

Definition 2 (zero controllability). System (2) is said to be zero controllable if any initial state sequence $x_{-j} \in \mathbb{R}_{+}^{n}$ $\left(j \in \sigma_{0}^{p}\right)$ and any initial input sequence $u_{-j} \in \mathbb{R}_{+}^{m}\left(j \in \sigma_{1}^{q}\right)$, there exist a positive integer $N$ and an input sequence $u_{i} \in \mathbb{R}_{+}^{m}\left(i \in \sigma_{0}^{N-1}\right)$ such that the state of the system is driven from $x_{-j}$ to 0 , that is, $x_{N}=0$.

The paper is organized as follows. In Section 2, we give and prove the criterion of the zero controllability of the general system (2) which is the main result of this paper. A numerical example is given in Section 3. Finally, the conclusion is provided in Section 4.

\section{Main Result}

In this section, we give the proof of the main result of this paper, which is accomplished in Theorem 1.

Lemma 2 (see $[12,13])$. The general solution to (2) is given by

$$
\begin{aligned}
x_{i}= & G_{i} x_{0}+\sum_{j=1}^{p} \sum_{k=1}^{p-j+1} G_{i-k} A_{k-1+j} x_{-j}+\sum_{j=1}^{q} \sum_{k=1}^{q-j+1} G_{i-k} B_{k-1+j} u_{-j} \\
& +\sum_{j=0}^{i-1} \sum_{k=0}^{q} G_{i-1-j-k} B_{k} u_{j}, \quad i \in \mathbb{N},
\end{aligned}
$$

where the transition matrix $G_{i} \in \mathbb{R}^{n \times n}(i \in \mathbb{N})$ is determined by the recurrence relation

$$
G_{i}= \begin{cases}I_{n}, & \text { for } i=0, \\ \sum_{k=0}^{p} A_{k} G_{i-1-k}, & \text { for } i \in \mathbb{N}^{*},\end{cases}
$$

with the assumption

$$
G_{i}=0, \quad \text { for } i<0 .
$$

Lemma 3. The transition matrix $G_{i}$ also satisfies the following equation:

$$
G_{i}=\sum_{k=0}^{p} G_{i-1-k} A_{k}, \quad \text { for } i \in \mathbb{N}^{*} .
$$

Then, for any $i \in \mathbb{N}$, we pose $H_{i}^{0}=G_{i}$, and hence, for all $i \in \mathbb{N}^{*}$, we pose

$$
\begin{cases}H_{i}^{j}=\sum_{k=1}^{p-j+1} H_{i-k}^{0} A_{k-1+j}, & j \in \sigma_{0}^{p}, \\ L_{i}^{j}=\sum_{k=1}^{q-j+1} H_{i-k}^{0} B_{k-1+j}, & j \in \sigma_{1}^{q},\end{cases}
$$

with

$$
\begin{gathered}
H_{i}^{j}=0, \quad \text { for } j \in \sigma_{1}^{p} \text { and } i \leq 0, \\
L_{i}^{j}=0, \quad \text { for } j \in \sigma_{1}^{q} \text { and } i \leq 0 .
\end{gathered}
$$

Moreover, for $i \in \mathbb{N}$, we put

$$
K_{i}=\sum_{k=0}^{q} H_{i-k}^{0} B_{k}
$$

with $K_{i}=0$ for $i<0$.

Clearly by (7) and (9), solution (3) is given by the following new formula:

$$
x_{i}=\sum_{j=0}^{p} H_{i}^{j} x_{-j}+\sum_{j=1}^{q} L_{i}^{j} u_{-j}+\sum_{j=0}^{i-1} K_{i-1-j} u_{j}, \quad i \in \mathbb{N} .
$$

Theorem 1. System (2) is zero controllable if and only if the matrix

$$
A=\left(\begin{array}{cccccccccc}
A_{0} & A_{1} & \cdots & \cdots & A_{p} & B_{1} & \cdots & \cdots & \cdots & B_{q} \\
I_{n} & 0 & \cdots & \cdots & 0 & 0 & \cdots & \cdots & \cdots & 0 \\
0 & \ddots & \ddots & & \vdots & \vdots & & & & \vdots \\
\vdots & \ddots & \ddots & \ddots & \vdots & \vdots & & & & \vdots \\
0 & \cdots & 0 & I_{n} & 0 & 0 & \cdots & \cdots & \cdots & 0 \\
0 & \cdots & \cdots & \cdots & 0 & 0 & \cdots & \cdots & \cdots & 0 \\
\vdots & & & & \vdots & I_{m} & \ddots & & & \vdots \\
\vdots & & & & \vdots & 0 & \ddots & \ddots & & \vdots \\
\vdots & & & & \vdots & \vdots & \ddots & \ddots & \ddots & \vdots \\
0 & \cdots & \cdots & \cdots & 0 & 0 & \cdots & 0 & I_{m} & 0
\end{array}\right)
$$

is nilpotent.

We introduce the following useful two lemmas that will aid us in the proof of our main result.

Lemma 4. For all $i \in \mathbb{N}$, we have

$$
\begin{aligned}
& \left\{\begin{array}{l}
H_{i+1}^{j}=H_{i}^{j+1}+H_{i}^{0} A_{j}, \quad j \in \sigma_{0}^{p-1}, \\
H_{i+1}^{p}=H_{i}^{0} A_{p},
\end{array}\right. \\
& \left\{\begin{array}{l}
L_{i+1}^{j}=L_{i}^{j+1}+H_{i}^{0} B_{j}, \quad j \in \sigma_{1}^{q-1}, \\
L_{i+1}^{q}=H_{i}^{0} B_{q} .
\end{array}\right.
\end{aligned}
$$


Without loss of generality, we assume that $p \geq q$. Indeed, if $p<q$, we can set $A_{j}=0$ for $j \in \sigma_{p+1}^{q}$, and then we come back to $p=q$ case.

Lemma 5. For all $i \geq p$, we have

$$
A^{i}=\left(\begin{array}{ccccccc}
H_{i}^{0} & H_{i}^{1} & \cdots & H_{i}^{p} & L_{i}^{1} & \cdots & L_{i}^{q} \\
H_{i-1}^{0} & H_{i-1}^{1} & \cdots & H_{i-1}^{p} & L_{i-1}^{1} & \cdots & L_{i-1}^{q} \\
\vdots & \vdots & & \vdots & \vdots & & \vdots \\
H_{i-p}^{0} & H_{i-p}^{1} & \cdots & H_{i-p}^{p} & L_{i-p}^{1} & \cdots & L_{i-p}^{q} \\
0 & \cdots & \cdots & 0 & 0 & \cdots & 0 \\
\vdots & & & \vdots & \vdots & & \vdots \\
0 & \cdots & \cdots & 0 & 0 & \cdots & 0
\end{array}\right) .
$$

Proof. See Appendix C.

Remark 1. Since $H_{p-p}^{0}=H_{0}^{0}=I_{n}$, then $A^{p} \neq 0$.

Now, we prove our main result.

Proof of Theorem 1 (sufficiency). Since $A$ is nilpotent, then there exists a positive integer $N$ such that $A^{N+p}=0$. Hence, by Lemma 5 , we have $H_{N}^{i}=0$ for $i \in \sigma_{0}^{p}$ and $L_{N}^{j}=0$ for $j \in \sigma_{1}^{q}$. Thus, system (2) is zero controllable.

(Necessity). Since system (2) is zero controllable, there exists a positive integer $N$ such that $H_{N}^{j}=0$ for $i \in \sigma_{0}^{p}$ and $L_{N}^{j}=0$ for $j \in \sigma_{1}^{q}$. According to Lemma 4, we get that $H_{N+k}^{j}=0$ and $L_{N+k}^{j}=0$ for $k \in \sigma_{0}^{p}$. Thus, by Lemma 5, we have $A^{N+p}=0$. This implies that $A$ is nilpotent. The theorem is proved.

Remark 2. If one diagonal element of the matrix $A_{0}$ is nonzero, system (2) is nonzero controllable.

\section{Example}

Consider system (2) with $p=q=2$ and matrices

$$
A_{0}=\left(\begin{array}{lll}
0 & 0 & 0 \\
0 & 0 & 0 \\
1 & 0 & 0
\end{array}\right),
$$$$
A_{1}=\left(\begin{array}{lll}
0 & 0 & 0 \\
0 & 0 & 0 \\
1 & 0 & 0
\end{array}\right),
$$$$
A_{2}=\left(\begin{array}{lll}
0 & 0 & 0 \\
2 & 0 & 0 \\
0 & 0 & 0
\end{array}\right),
$$

$$
B_{1}=\left(\begin{array}{l}
0 \\
1 \\
0
\end{array}\right) \text {, }
$$

$$
B_{2}=\left(\begin{array}{l}
0 \\
0 \\
2
\end{array}\right) \text {. }
$$

By calculation, we get that matrix $A=$ $\left(\begin{array}{ccccc}A_{0} & A_{1} & A_{2} & B_{1} & B_{2} \\ I_{3} & 0 & 0 & 0 & 0 \\ 0 & I_{3} & 0 & 0 & 0 \\ 0 & 0 & 0 & 0 & 0 \\ 0 & 0 & 0 & I_{1} & 0\end{array}\right)$ is nilpotent with index $k=6$, that is, $A^{k-1} \neq 0$ and $A^{k}=0$. Thus, by Theorem 1 , system (2) is zero controllable.

\section{Conclusion}

In this paper, we have investigated the zero controllability of discrete linear positive systems with delays. Necessary and sufficient conditions have been established for the zero controllability discrete linear positive systems with multiple delays in both state variables and input signals. A numerical example is presented to explore the proposed theory. 
Appendix

For $i \in \sigma_{1}^{p}$, we have

\section{A. Proof of Lemma 3}

Proof. First, for $i=1$, we have $G_{1}=A_{0}=\sum_{k=0}^{p} G_{-k} A_{k}$ and (6) holds. Secondly, suppose that (6) holds for $k \in \sigma_{1}^{i}$. We prove that it holds for $k=i+1$.

$$
\begin{aligned}
G_{i+1} & =\sum_{k=0}^{i} A_{k} G_{i-k}=\sum_{k=0}^{i-1} A_{k} G_{i-k}+A_{i}=\sum_{k=0}^{i-1} A_{k}\left(\sum_{j=0}^{p} G_{i-k-1-j} A_{j}\right)+A_{i} \\
& =\sum_{k=0}^{i-1} A_{k}\left(\sum_{j=0}^{i-k-1} G_{i-k-1-j} A_{j}\right)+A_{i}=\sum_{j=0}^{i-1}\left(\sum_{k=0}^{i-j-1} A_{k} G_{i-j-1-k}\right) A_{j}+A_{i} \\
& =\sum_{j=0}^{i-1} G_{i-j} A_{j}+A_{i}=\sum_{j=0}^{i} G_{i-j} A_{j}=\sum_{j=0}^{p} G_{i-j} A_{j} .
\end{aligned}
$$

For $i \geq p+1$, we have

$$
\begin{aligned}
G_{i+1} & =\sum_{k=0}^{p} A_{k} G_{i-k}=\sum_{k=0}^{p} A_{k}\left(\sum_{j=0}^{p} G_{i-k-1-j} A_{j}\right) \\
& =\sum_{j=0}^{p}\left(\sum_{k=0}^{p} A_{k} G_{i-j-1-k}\right) A_{j}=\sum_{j=0}^{p} G_{i-j} A_{j} .
\end{aligned}
$$

Thus, (6) is satisfied in step $i+1$. Hence, (6) holds for any $i \in \mathbb{N}^{*}$.

\section{B. Proof of Lemma 4}

Proof. Let $i \in \mathbb{N}$. For $j \in \sigma_{0}^{p-1}$, we have

$$
\begin{aligned}
H_{i+1}^{j}-H_{i}^{j+1} & =\sum_{k=1}^{p-j+1} H_{i+1-k}^{0} A_{k-1+j}-\sum_{k=1}^{p-j} H_{i-k}^{0} A_{k+j} \\
& =\sum_{k=0}^{p-j} H_{i-k}^{0} A_{k+j}-\sum_{k=1}^{p-j} H_{i-k}^{0} A_{k+j} \\
& =H_{i}^{0} A_{j},
\end{aligned}
$$

and, for $j=p$, we have

$$
H_{i+1}^{p}=\sum_{k=1}^{1} H_{i+1-k}^{0} A_{k-1+p}=H_{i}^{0} A_{p} .
$$

Similarly, we prove that (13) holds.

\section{Proof of Lemma 5}

Proof. We introduce a new state variable $\tilde{x}_{i} \in \mathbb{R}^{n(p+1)+m q}$ for $i \in \mathbb{N}$ by

$$
\tilde{x}_{i}=\left(\begin{array}{c}
x_{i} \\
x_{i-1} \\
\vdots \\
x_{i-p} \\
u_{i-1} \\
\vdots \\
u_{i-q}
\end{array}\right) .
$$

It is easy to verify that

$$
\left\{\begin{array}{l}
\tilde{x}_{i+1}=A \tilde{x}_{i}+B u_{i}, \quad i \in \mathbb{N}, \\
\tilde{x}_{0} \in \mathbb{R}^{n(p+1)+m q},
\end{array}\right.
$$

where $A$ is defined in (11) and

$$
B=\left(\begin{array}{c}
B_{0} \\
0 \\
\vdots \\
0 \\
I_{m} \\
0 \\
\vdots \\
0
\end{array}\right) .
$$

Let $u_{i}=0$ for $i \in \mathbb{N}$. Then, the solution of system (C.2) is given by

$$
\widetilde{x}_{i}=A^{i} \widetilde{x}_{0}, \quad i \in \mathbb{N} .
$$

On the other hand, from (10), for all $i \geq p$, we have 


$$
\tilde{x}_{i}=\left(\begin{array}{c}
x_{i} \\
x_{i-1} \\
\vdots \\
x_{i-p} \\
0 \\
\vdots \\
0
\end{array}\right)=\left(\begin{array}{ccccccc}
H_{i}^{0} & H_{i}^{1} & \cdots & H_{i}^{p} & L_{i}^{1} & \cdots & L_{i}^{q} \\
H_{i-1}^{0} & H_{i-1}^{1} & \cdots & H_{i-1}^{p} & L_{i-1}^{1} & \cdots & L_{i-1}^{q} \\
\vdots & \vdots & & \vdots & \vdots & & \vdots \\
H_{i-p}^{0} & H_{i-p}^{1} & \cdots & H_{i-p}^{p} & L_{i-p}^{1} & \cdots & L_{i-p}^{q} \\
0 & \cdots & \cdots & 0 & 0 & \cdots & 0 \\
\vdots & & & \vdots & \vdots & & \vdots \\
0 & \cdots & \cdots & 0 & 0 & \cdots & 0
\end{array}\right)\left(\begin{array}{c}
x_{0} \\
x_{-1} \\
\vdots \\
x_{-p} \\
u_{-1} \\
\vdots \\
u_{-q}
\end{array}\right) .
$$

Hence, by identification between (C.4) and (C.5), we get that (14) holds.

\section{Data Availability}

No data were used to support this study.

\section{Conflicts of Interest}

The author declares no conflicts of interest.

\section{References}

[1] L. Farina and S. Rinaldi, Positive Linear Systems: Theory and Applications, J. Wiley \& Sons, New York, NY, USA, 2000.

[2] T. Kaczorek, Positive $1 D$ and 2D Systems. Communications and Control Engineering, Springer-Verlag, London, UK, 2002.

[3] D. G. Luenberger, Introduction to Dynamic Systems: Theory, Models and Applications, Academic Press, New York, NY, USA, 1979.

[4] A. Berman and R. J. Plemmons, Nonnegative Matrices in the Mathematical Sciences, Vol. 9, Society for Industrial and Applied Mathematics, Philadelphia, PA, USA, 1994.

[5] D. N. P. Murthy, "Controllability of a linear positive dynamic system," International Journal of Systems Science, vol. 17, no. 1, pp. 49-54, 1986.

[6] P. G. Coxson and H. Shapiro, "Positive input reachability and controllability of positive systems," Linear Algebra and Its Applications, vol. 94, pp. 35-53, 1987.

[7] V. G. Rumchev and D. J. G. James, "Controllability of positive linear discrete-time systems," International Journal of Control, vol. 50, no. 3, pp. 845-857, 1989.

[8] M. P. Fanti, B. Maione, and B. Turchiano, "Controllability of multi-input positive discrete-time systems," International Journal of Control, vol. 51, no. 6, pp. 1295-1308, 1990.

[9] L. Caccetta and V. G. Rumchev, "A survey of reachability and controllability for positive linear systems," Annals of Operations Research, vol. 98, no. 1/4, pp. 101-122, 2000.

[10] T. Kaczorek and M. Busowicz, "Reachability and minimum energy control of positive linear discrete-time systems with multiple delays in state and control," Pomiary Automatyka Kontrola, vol. 53, no. 10, pp. 40-44, 2007.
[11] G. Xie and L. Wang, "Reachability and controllability of positive linear discrete-time systems with time-delays," in Positive systems (Rome, L. Benvenuti, A. De Santis, and L. Farina, Eds., vol. 294, pp. 377-384, Springer, Berlin, Germany, 2003.

[12] M. Naim, F. Lahmidi, A. Namir, and M. Rachik, "On the output controllability of positive discrete linear delay systems," Abstract and Applied Analysis, vol. 2017, Article ID 3651271, 12 pages, 2017.

[13] M. Busłowicz, "On some properties of the solution of state equation of discrete-time systems with delays," Zesz. Nauk. Polit. Bial., Elektrotechnika, vol. 1, pp. 17-29, 1983, in Polish. 